\title{
Model for Trans-sector Digital Interoperability
}

\author{
António Madureira ${ }^{1}$, Frank den Hartog $^{2}$, Eduardo Silva ${ }^{3}$ and Nico Baken ${ }^{1,4}$ \\ 1 Network Architectures and Services (NAS) group, Delft University of Technology, \\ Delft, The Netherlands \\ 2 TNO, Delft, The Netherlands \\ 3 Centre for Telematics and Information Technology (CTIT), University of Twente, \\ Enschede, The Netherlands \\ 4 Corporate Strategy and Innovation department, Royal KPN, The Hague, The \\ Netherlands
}

\begin{abstract}
Interoperability refers to the ability of two or more systems or components to exchange information and to use the information that has been exchanged. The importance of interoperability has grown together with the adoption of Digital Information Networks (DINs). DINs refer to information networks supported by telecommunication infrastructures and terminated by microprocessors. With an upcoming interest in services science and transsector business models, a stronger driver arises to further break the interoperability barriers across sectors. In this paper, we propose a novel model to address trans-sector digital interoperability, which by definition involves interoperability across different economic sectors connected by DINs. Particularly, we specify how a well known interoperability framework, the ATHENA framework, should be adapted for the economic sector plane. Based on data from the Eurostat survey on ICT usage and e-Commerce in enterprises, we illustrate how conclusions about trans-sector interoperability can be extracted and technological implications can be derived.
\end{abstract}

Keywords: interoperability; MDA; sector; economic; model; productivity; service science; ATHENA; digital information network

\subsection{Introduction}

IT systems interoperability is a growing interest area, mainly motivated by the need of integrating new, legacy and evolving systems [5]. Interoperability refers to the "ability of two or more systems or components to exchange information and to use the information that has been exchanged" [12]. The importance of interoperability has increased together with the adoption of Digital Information Networks (DINs). DINs refer to information networks supported by 
telecommunication infrastructures and terminated by microprocessors. DINs support the digital economy: an economy that is based on digital goods and services in any of the production, distribution and consumption stages.

According to the ATHENA framework [5], interoperability takes place, at least, at four levels: business, process, service and information. In this paper, we investigate trans-sector digital interoperability, which by definition involves interoperability across different economic sectors connected through DINs. We define sector as a cluster of organisations performing homogeneous activities.

Sectors such as Healthcare, Education or Transport have different societal roles [1]. Therefore, enterprises from different sectors are inherently not competing in the same market, since they provide different products or services. Besides sectors operating in the same traditional value chains, the invisible hand of the markets does not lead enterprises to look across the boundaries of their own sector. With an upcoming interest in services science [20] and novel trans-sector business models [2], sometimes with more intangible social or environmental outcomes (e.g. eGovernment), a stronger driver arises to break the interoperability barriers across sectors.

Bastiaansen and Baken [3] conceptualise two approaches for trans-sector interoperability: 1) top-down interoperability: the functionality to be realised must be leading in the IT system development process. Thus, the business requirements must be top-down translated into trans-sector implementations. A method for this approach is Model Driven Architecture (MDA) (e.g. the ATHENA framework); 2) bottom-up interoperability: trans-sector processes require the integration of the existing functions provided by the individual sectors. Such integration requires rich and unambiguous syntactic and semantic description of the IT functions provided by the sectors. A method for this approach is Semantic Web Services (SWS).

The ATHENA framework gives a good starting point for designing a top-down interoperability model, but it should be taken from the enterprise plane to the economic sector plane. In this paper, based upon the ATHENA framework, we propose a novel model to address trans-sector digital interoperability. Particularly, we specify how the ATHENA framework should be adapted for the economic sector plane. With this model, we follow the top-down interoperability approach principle of identifying the business requirements before translating them into trans-sector implementations. Naturally, the model described is also useful in a bottom-up interoperability approach, because it helps to define syntactic and semantic descriptions of IT systems, potentially with direct business value. To validate the usefulness of the model, we describe how to derive technological implications from the application of the model.

This paper is organised as follows. In section 1.2, we describe the state of the art regarding trans-sector digital interoperability. In section 1.3, we introduce a set of mechanisms that serves as the base for the model proposed in this paper. In section 1.4, we provide an overview of the complete model. Finally, in section 1.5, we draw some conclusions and indicate directions for future work. 


\subsection{State of the art}

Traditionally, scientific research on IT interoperability is a bottom-up process. Examples of this approach are semantic web service frameworks such as IRS-II [23], OWL-S [26] and WSMF [10]. Compared to IRS-II and OWL-S, WSMF takes a more business-oriented approach, focusing on a set of e-commerce requirements for Web services. But in practice, it lacks a top-down view of business requirements to make the components it describes (ontologies, goal repositories and mediators) more concrete for business purposes. Bottom-up semantic interoperability already evolved to standardisation efforts [13]. Motivation for this research comes from Virtual Enterprises (VE), Virtual Organisations (VO), Computer Supported Collaborative Work (CSCW), Workflow Management System (WFMS), Process-Centred Environments (PCEs), etc.

ATHENA [5], a recent European project on interoperability, attempted a topdown approach. The uniqueness of this project lies in its multidisciplinary character, merging three research areas: 1) enterprise modelling to identify business requirements; 2) architectures and platforms to define implementation frameworks; and 3) ontologies to identify interoperability semantics.

Figure 1.1 presents the ATHENA reference model indicating the required and provided artefacts of two connected enterprises. Interoperation can take place at various levels (enterprise/business, process, service and information/data). For each of these levels, a model-driven interoperability approach is prescribed where models are used to formalise and exchange the provided and required artefacts that must be negotiated and agreed upon.

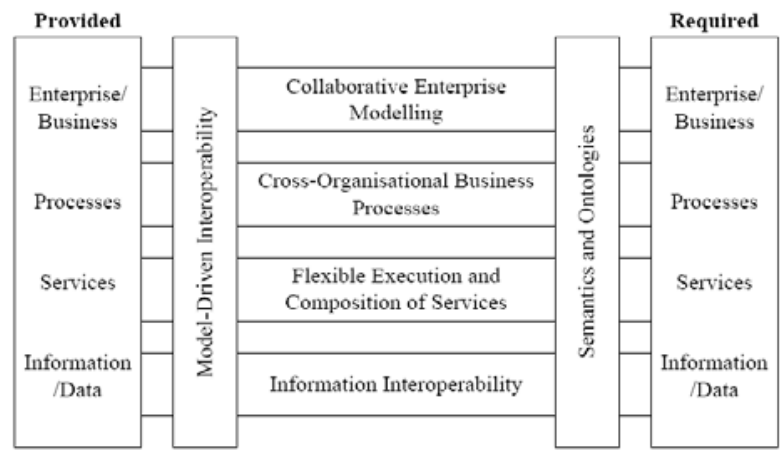

Fig. 1.1 ATHENA reference model

One ATHENA meta model is particularly relevant to our work: the Crossorganisational Business Process (CBP) model. The CBP tries to capture tasks and relationships from the different parties involved in cross-organisational business processes. One example of a process is a retailer-manufacturer cooperation: 1) a supplier sends a request for quotation to the manufacturer; 2) the manufacturer checks the stock; and 3) the manufacturer accepts the quotation and responds with an order. 
To our knowledge, no one has yet took the effort of breaking the meta concept of process into more refined terms, particularly at a general and complete, but necessarily abstract, economic plane. Here lies the value and originality of our work: breaking the concept of process at an economic trans-sector plane to be used in an interoperability framework as the ATHENA. The novelty of our trans-sector approach for interoperability can be verified by a conclusion taken in [19]: the theoretical and empirical support for the relation between DINs and economic productivity is still inconclusive. Even economists focusing on understanding the economic importance of DINs, thus DINs' business value, still struggle to clarify it. In [19], a framework contributing to clarify this relation has been laid down which serves as the base for our trans-sector interoperability model described in this paper.

Other projects have taken further action to meet the recognised need to clarify the concept of process for deriving technological requirements. Pratl et. al [27] introduce an eighth layer in the OSI model defining profiles for (manufacturing) control purposes. Bauer et. al [4] extend the OSI model with human factors, providing a conceptual tool to facilitate the discussions in human-computerinteraction disciplines.

\subsection{Capabilities}

Productivity refers to a summarised measure of performance (P), based on the ratio of the total value of output $(\mathrm{O})$ divided by the total value of input (I) (see [15]): $\mathrm{P}=\mathrm{O} / \mathrm{I}$. In this section, we describe a set of mechanisms (defined as capabilities) which take an economic agent using DINs to a higher level of productivity. Any mechanism incrementing productivity leads to business value. Unlike mere operational functions (e.g. routing, forwarding, transferring, etc), the mechanisms identified are part of the interface between business and service. These mechanisms are generally applicable to economic agents across all sectors. Thus, they are the mechanisms required to identify a model for trans-sector digital interoperability. Before describing this set of mechanisms, we start by referring to the concept of externality to introduce a definition of economic agent. From here, follows a definition of capability, which is our conceptualisation of a direct causal mechanism linking DINs to sectoral productivity.

Network externality can be defined as a change in productivity that an individual achieves when the number of other individuals using DINs changes. This allows, in principle, to separate the value of productivity into two distinct parts. One component, the autarky value, is the productivity value if there are no other individuals using DINs. The other component, the connection value, is the additional productivity value achieved when multiple other individuals are using DINs. The latter value is the essence of DINs' externality effects. Using the definition of connection value, we define economic agent in the following way: an economic agent is any entity from an economic environment which may achieve an additional productivity value when multiple other individuals are using DINs. 
Examples of agents are researchers using DINs to search for knowledge and companies marketing their products on-line. An agent explores personal and intrinsic capabilities to become more productive within his economic environment. For example, consumer A meets supplier B to acquire a production input at a lower price. The capability of A and B to meet each other will make both more productive. From a thorough literature review on the relation between information, digital infrastructures and productivity, we have come across time and time again with a relevant set of six capabilities of a productive economic agent, which are directly dependent on DINs and impact productivity. We define capability as: capability is a quality of the economic agent used for productive purposes and directly effected by DINs. In the following subsections we describe these six capabilities, which are generally applicable to agents across all economic sectors.

\subsubsection{Coordinativity}

Coordination is "the act of managing interdependencies between activities performed to achieve a goal" (see [21]). It arises, effecting productivity, when the agent has to choose between actions, the order of the actions matters and/or the time at which the actions are carried out matters. This leads to: coordinativity is the capability of an economic agent to manage interdependencies between activities with other agents to jointly achieve a common goal.

Coordinativity prevents conflicts, waste of efforts, and squandering resources, and assures focus, while trying to accomplish a common goal. The work of Kandori et. al [16] has triggered much interest in coordination games. Important research results concern the impact of different network structures in coordination. In a survey, $45 \%$ of the respondents identified DINs as a driver to reorganise work practices (see [14]). More specifically, on-line remote monitoring can be seen as a good example of an application of digital coordination.

\subsubsection{Cooperativity}

Cooperation can be defined as "acting together with a common purpose" (see [11]). Sharing information helps agents aligning their individual incentives with outcomes. Assuming proper behaviour, if absolute incentives are more advantageous over relative incentives, the agents cooperate. Both inter- and intraorganisational cooperation have been object of study since the work of Marshall [22]. Good examples are joint ventures. This leads to: cooperativity is the capability of an economic agent to align his personal goals with different individual goals from other agents for a common purpose.

In practice, it is often hard to distinguish cooperativity from coordinativity. Conceptually, the main differences are: 1) in coordinativity the agents share exactly the same goals, while in cooperativity the agents share only partially aligned goals; and 2) in coordinativity the relation between the agents is critically dependent on time, while in cooperativity the agents relate to each other typically off-line. Although the experimental literature on cooperation is vast, only a few papers consider the role of networks in this process (see e.g. [31]). Supply and 
demand matching with on-line trading is an important practical example of the importance of DINs for cooperativity.

\subsubsection{Adoptativity}

Nelson and Winter [24] state that firms improve their productivity by adopting technological and organisational solutions from the most innovative firms. Examples are informal associations and product advertisement. Important dimensions to be accounted are the level of codification and the extent to which the knowledge fits in a set of interdependent elements. This leads to: adoptativity is the capability of an economic agent to adopt knowledge from other agents.

There is a vast literature studying adoptativity using network analysis. It started with Ryan and Gross [28] studying adoption of pesticides by rural sociologists, and Coleman et. al [7] studying the adoption of medicines. Many examples could be cited showing the value of digital networks to exchange knowledge. A good example is e-learning between students.

\subsubsection{Creativity}

Agents can increase their productivity by creating new knowledge following from collaborating with other agents to address operational inefficiencies. Their motivation to collaborate comes from their limited specialised knowledge and changes in their environment. Organisations that best address crucial information gaps through their information network structures may be more able to create novel knowledge. This leads to: creativity is the capability of an economic agent to create new knowledge, unknown to him before and to his collaborative agents.

The relevance of DINs for collaborative research is well recognised (see [25]), and evidences have been found that organisations that use them more intensively, innovate more (see [17]). A trade-off exists between the rate of information gathering and the rate of environmental change. A good example of creativity is research in universities.

\subsubsection{Selectivity}

Selection is the process of scanning for the unknown or generating courses of action that improve on known alternatives (see [6]). For maximal productivity, the agent has to decide for a stopping point in an uncertain environment, while keeping computational requirements within limits. This leads to: selectivity is the capability of an economic agent to scan and value information from other agents, generating courses of action that improve on known alternatives.

The role of information networks has been extensively acknowledged in this process (see [32]). A practical proposal accounting the value of networks in the process of selection has been made by Saaty [29]. This framework has been used for interdependent information system project selection. On-line job hunting and Google.com are good examples of selectivity using DINs. 


\subsubsection{Negotiability}

Negotiability occurs when exchange happens between unfamiliar partners or when evaluating new courses of action. Negotiation grows in importance with the perception that potential downside effects of a wrong decision can be large and costly to reverse. Negotiability mechanisms include signalling (e.g. give guarantees to buy) and screening (e.g. give certificates to sell). Economic literature further distinguishes between one shot and repeated contracts. This leads to: negotiability is the capability of an economic agent to bargain with other agents for lower exchange costs.

Kranton and Minehart [18] developed a model in which the prices are determined by a bargaining process rather than an English auction. However, the precise influence of the network structure in negotiation processes has not been intensively studied yet. On-line stock trading activities are a good example of the importance of DINs for negotiability.

\subsection{Trans-sector interoperability model}

\begin{tabular}{|c|c|c|}
\hline Sector A & \multirow{2}{*}{$\begin{array}{l}\text { Trans-sector } \\
\text { Interoperability } \\
\text { Productivity }\end{array}$} & Sector B \\
\hline Business & & Business \\
\hline \multirow{6}{*}{$\begin{array}{l}\text { Capabilities/ } \\
\text { Processes }\end{array}$} & Creativity & \multirow{6}{*}{$\begin{array}{r}\text { Capabilities/ } \\
\text { Processes }\end{array}$} \\
\hline & Adoptativity & \\
\hline & Cooperativity & \\
\hline & Coordinativity & \\
\hline & Negotiability & \\
\hline & Selectivity & \\
\hline Service & \multirow{2}{*}{ DINs } & Service \\
\hline Sector A & & Sector B \\
\hline
\end{tabular}

Fig. 1.2 Trans-sector interoperability model

Figure 1.2 resumes our model for trans-sector interoperability. Naturally, it is based upon the ATHENA model of figure 1.1, but specifies the general processes for the trans-sector (economic) plane. The processes are the capabilities referred in the previous section. In this context, it makes more sense to talk about inter-sector interactions rather than inter-enterprise interactions. Naturally, being sectors clusters of enterprises, these capabilities are also applicable to inter-enterprise interactions.

The scheme represents the four layers of sectors A and B: DINs and other IT (e.g. computer terminals), service, processes and business. The middle part shows the various capabilities/processes analysed in this paper, symbolising how exploiting these capabilities increases productivity when DINs are used for interaction between sectors. The capabilities are shown in random order. In the future, however, we would like to input some structure to the order of the 
capabilities. Intuitively, we would expect productivity to be more sensible to some of these capabilities. For example, creativity is a source for innovations which might have a profound effect on productivity. Selectivity, on the other hand, seems to be an input to other capabilities (e.g. adoptativity). Therefore, it might be more complicated to correlate data on selectivity with data on productivity.

DINs are the infrastructure which enables the economic agents to use these capabilities to increase their productivity, regardless of the specific sectors they are dealing with on a case to case basis. These capabilities are generally applicable to agents across all economic sectors. Therefore, at an aggregated macro level, our model may help to manage and control trans-sector interoperability, productivity, and innovation on a national or even global scale from a capabilities point of view.

Figure 1.3 is an attempt to quantify the importance of coordinativity and adoptativity for different economical sectors. The data source used is the Eurostat survey on ICT usage and e-Commerce in enterprises [9]. The amount of data is quite significant, spanning the years from 2002 to 2008, various countries from EU, with regional and sectoral breakdowns, for a large collection of different aspects related to the use of ICT in enterprises. For this paper, only data from 2007 for the Netherlands has been used. For both subfigures, the proxy variable used for DINs is "have access to Internet" (reference is e_iacc). For coordinativity, the proxy variable used is "use of systems for managing production, logistics or service operations (reference is $e_{-} l n k p l s$ ). For adoptativity, the proxy variable is "purpose of the Internet (as a customer): training and education" (reference is e_iedu). The numerical references in the figures (e.g. $10+: 72$ ) refer to more refined aggregations of enterprises (e.g. a particular type of manufacturing enterprises).
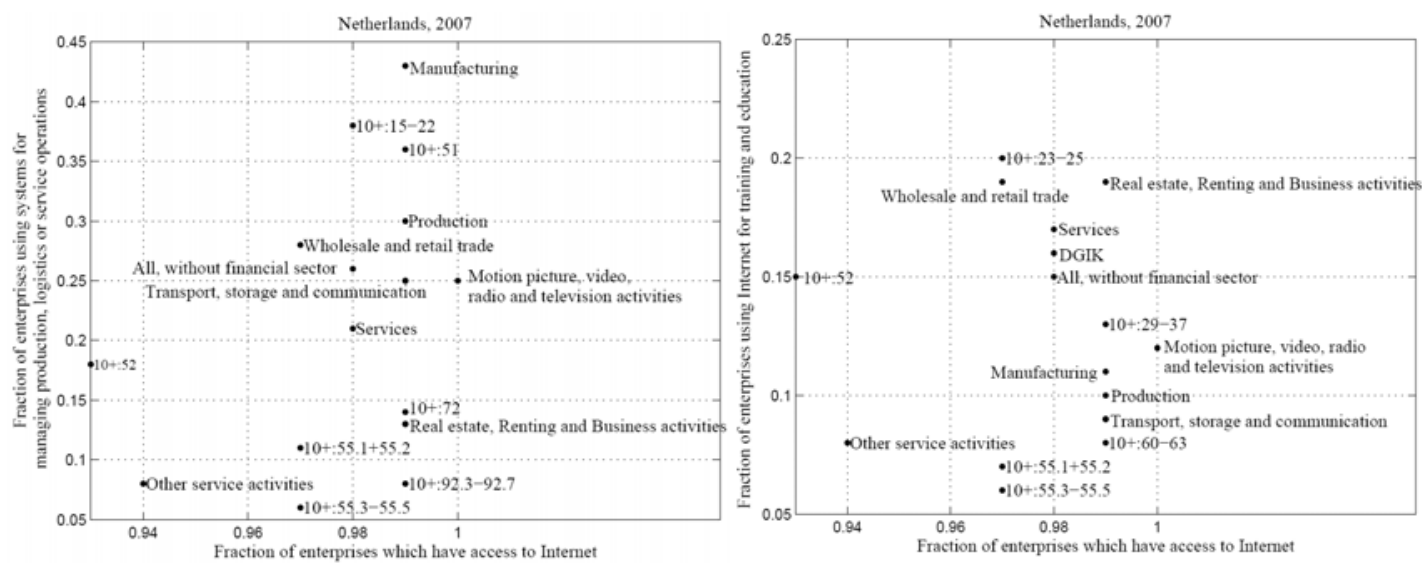

Fig. 1.3 Importance of coordinativity (left) and adoptativity (right) per sector

The limitations of the data only allow us to take preliminary and exemplifying conclusions. For example, coordinativity seems to be more important for the manufacturing sector than for real estate, renting and business activities sector. The 
inverse could be said for adoptativity: the manufacturing sector performs less online training and education than the real estate, renting and business activities sector. These figures could be considerably improved at least in three ways: 1) the Eurostat only collects data for particular economic sectors (e.g. manufacturing). Other sectors are not observed (e.g. the education sector). For a full overview of trans-sector interoperability, all the sectors should be evaluated; 2) the sector classification used is the Classification of Economic Activities in the European Community (NACE). Some sectors classified are hardly understandable (e.g. the "production" sector). There is no worldwide consensus is any classification, and furthermore, classifications evolve over time as the relevance of specific activity clusters varies. A proper sector classification has to be used that discriminates sectors in a clear and relevant way. Worldwide, the UN International Standard Industrial Classification (ISIC) [30] classification seems to be the preferred one; and 3) the proxies used for the capabilities should be composed measures of various available data source variables. E.g. coordinativity could also have been measured with the variable "use invoicing and paying systems" (reference is e_lnkpay).

After understanding which capabilities are more relevant for the different sectors, a survey to IT departments of representative enterprises of the different sectors could be done to investigate which IT applications are commonly used to address those capabilities (e.g. online agenda applications for coordinativity). Finally, these IT applications should be analysed concerning the fulfilment of interoperability requirements (e.g. use of open standards). Hence, conclusions could be extracted concerning the status of trans-sector interoperability. Furthermore, technological requirements for those IT applications could be derived to increase trans-sector interoperability. The procedure described in the previous paragraph will be used in our future work.

\subsection{Conclusion and future work}

Generally, we conclude that current interoperability architectural models do not take a full top-down approach when translating business requirements into technological implementations. The ATHENA model gives a good starting point for designing such a top-down interoperability model, but it should be taken from the enterprise plane to the economic sector plane. One of the model's levels requiring adaptation is the processes level.

To address the economic plane of interoperability, we propose a trans-sector interoperability model based upon a set of mechanisms (defined as capabilities) which take an economic agent using DINs to a higher level of productivity. Any mechanism incrementing productivity leads to business value. Unlike mere operational functions (e.g. routing, forwarding, transferring, etc), the mechanisms identified are part of the interface between business and service. These mechanisms are generally applicable to economic agents across all sectors. Thus, they are the mechanisms required to identify a model for trans-sector digital interoperability. Using data from the Eurostat survey on ICT usage and eCommerce in enterprises, we have made a preliminary attempt to quantify the 
importance of two capabilities (coordinativity and adoptativity) for different economical sectors. Although their relative relevance and orthogonality should still be investigated, from our thorough literature review, we are confident to state that these capabilities most probably form a complete set. However, it should be noted that we only took economic capabilities into account. Human and social factors are also important, but are not yet included. Our future work goes along six directions: 1) the capabilities described in this paper are used by an economic agent to rationally navigate through a production space problem. However, other factors are affected by DINs that, indirectly, affect productivity, particularly human (e.g. limitations in information sensing) and social factors (e.g. trust). Thus, further development of the model is required to include these factors; 2) we aim to functionally decompose the capabilities of the model to test if they overlap. From here, commonalities between the capabilities might be revealed; 3) further integration of the model with the top-down interoperability framework ATHENA; 4) perform a thorough analysis to understand which capabilities are more relevant for the different sectors; 5) perform a survey to representative enterprises from different sectors to understand which IT applications are commonly used to address the capabilities, and analyse these IT applications concerning the fulfilment of interoperability requirements; and 6) extract conclusions about transsector interoperability and derive technological requirements for IT applications to increase trans-sector interoperability.

\subsection{References}

[1] N.H.G. Baken, N. van Belleghem, E. van Boven, and A. de Korte. Unravelling 21st Century Riddles -Universal Network Visions from a Human Perspective. The Journal of The Communication Network, 5(4):11- 20, 2007.

[2] N.H.G. Baken, E. van Boven, and A.J.P.S. Madureira. Renaissance of the Incumbents: Network Visions from a Human Perspective. eChallenges 2007 Conference, The Hague, The Netherlands, October 2007.

[3] H.J.M. Bastiaansen and N.H.G. Baken. Using Advanced Integration Concepts for Trans-sector Innovation- View and Status. In Proceedings of the FITCE Conference, 2007.

[4] J.M. Bauer, P. Gai, J. Kim, T.A. Muth, and S.S. Wildman. Broadband: Benefits and Policy Challenges. Technical report, Quello Center for Merit Network Inc., 2002.

[5] A. Berre, B. Elvesæter, N. Figay, C. Guglielmina, S. Johnsen, D. Karlsen, T. Knothe, and S. Lippe. The ATHENA Interoperability Framework. In Enterprise Interoperability II: New Challenges and Approaches, pages 569-580. Springer, 2007.

[6] N. Bulkley and M. Van Alstyne. Why Information Should Influence Productivity. Working Paper Series 202, MIT Sloan School of Management, 2004.

[7] J.S. Coleman, E. Katz, and H. Menzel. Medical Innovation: A Diffusion Study. New York: Bobbs-Merrill, 1966.

[8] F. den Hartog, M. Blom, C. Lageweg, M. Peeters, J. Schmidt, R. van der Veer, A. de Vries, M. van der Werff, Q. Tao, R. Veldhuis, N. Baken, and F. Selgert. First experiences with Personal Networks as an enabling platform for service providers. In Proceedings of the Second Workshop on Personalized Networks, 2007.

[9] Eurostat. ICT usage and e-Commerce in enterprises. http://epp.eurostat.ec.europa.eu/portal/page/portal/information, 2009. 
[10] D. Fensel and C. Bussler. The Web Service Modeling Framework WSMF. Electronic Commerce Research and Applications, 1(2):113-137, 2002.

[11] Z. Hua. Study of Multi-Agent Cooperation. In Proceedings of Third International Conference on Machine Learning and Cybernetics. IEEE, 2004.

[12] IEEE. IEEE standard computer dictionary : a compilation of IEEE standard computer glossaries. IEEE Computer Society Press, 1991.

[13] ISO. Industrial automation systems and integration- Manufacturing software capability profiling for interoperability. Standard 16100, ISO, 2002.

[14] P. James and P. Hopkinson. Sustainable broadband? The Economic, Environmental and Social Impacts of Cornwall's actnow Project. Technical report, University of Bradford and SustainIT, 2005.

[15] D.W. Jorgenson and Z. Griliches. The Explanation of Productivity Change. Review of Economic Studies, 34:349-383, 1967.

[16] M. Kandori, G.J. Mailath, and R. Rob. Learning, Mutation, and Long Run Equilibria in Games. Econometrica, 61(1):29-56, 1993.

[17] P. Koellinger. Impact of ICT on corporate performance, productivity and employment dynamics. Technical Report Industry, Special Report No. 01/2006, e-Business Watch, European Commission and DG Enterprise, 2006.

[18] R. Kranton and D.F. Minehart. A Theory of Buyer-Seller Networks. American Economic Review, 91(3):485-508, 2001.

[19] A. Madureira, N. Baken, and H. Bouwman. Towards a Framework to Analyze Causal Relations From Digital Information Networks To Micro Economic Productivity. World Congress on the Knowledge Society, Venice, Italy, 2009.

[20] P. Maglio and J. Spohrer. Fundamentals of service science. Journal of the Academy of Marketing Science, 36(1):18-20, 2008.

[21] T.W. Malone and K. Crowston. What is coordination theory and how can it help design cooperative work systems? In CSCW '90: Proceedings of the 1990 ACM conference on Computer-supported cooperative work, pages 357-370. ACM Press, 1990.

[22] A. Marshall. Principles of Economics. London: Mcmillan, 1890.

[23] E. Motta, J. Domingue, L. Cabral, and M. Gaspari. IRS-II: A Framework and Infrastructure for Semantic Web Services. In The SemanticWeb - ISWC 2003, pages 306-318. Springer Berlin/Heidelberg, 2003.

[24] R.R. Nelson and S.G. Winter. Evolutionary Theory of Economic Change. Belknap Press, 1985.

[25] OECD. OECD Broadband Portal. http://www.oecd.org/sti/ict/broadband, 2008.

[26] OWL-S Coalition. OWL-S 1.0 Release. Website, 2003. http://www.daml.org/services/owl-s/1.0.

[27] G. Pratl, D. Dietrich, G. Hancke, and W. Penzhorn. A New Model for Autonomous, Networked Control Systems. Industrial Informatics, IEEE Transactions on, 3(1):2132, 2007.

[28] B. Ryan and N.C. Gross. The Diffusion of Hybrid Seed Corn in Two Iowa Communities. Rural Sociology, 23:15-24, 1943.

[29] T.L. Saaty. The Analytic Network Process: Decision Making with Dependence and Feedback. RWS Publications, 2001.

[30] UN. International Standard Industrial Classification of all Economic Activities (ISIC) Revision 3.1. Statistical Paper ST/ESA/STAT/SER.M/4/Rev.3.1, United Nations, 2002.

[31] F. Vega-Redondo. Building Up Social Capital in a Changing World. Discussion paper, University of Alicante, 2002.

[32] D. J. Watts, P. S. Dodds, and M. E. J. Newman. Identity and Search in Social Networks. Science, 296:1302, 2002. 